\begin{tabular}{l}
\hline International Journal of Mathematics and Computer Research \\
ISSN: $2320-7167$ \\
Volume 09 Issue 05 May 2021, Page no. $-2293-2302$ \\
Index Copernicus ICV: 57.55 , Impact Factor: 7.184 \\
DOI: $10.47191 /$ ijmcr/v9i5.03
\end{tabular}

\title{
Estimation of Scale Parameter of Weibull-Lomax Distribution via Bayesian Approach
}

\author{
Arun Kumar Rao, Himanshu Pandey \\ Department of Mathematics \& Statistics DDU Gorakhpur University, Gorakhpur, INDIA
}

\section{Abstract}

In this paper, the Weibull-Lomax distribution is considered for Bayesian analysis. The expressions for Bayes estimators of the parameter have been derived under squared error, precautionary, entropy, K-loss, and Al-Bayyati's loss functions by using quasi and gamma priors.

Keywords :Bayesian method, Weibull-Lomax distribution, quasi and gamma priors, squared error, precautionary, entropy, K-loss, and Al-Bayyati's loss functions.

\section{Introduction}

The Weibull-Lomax distribution has been proposed by Tahir et al. [1]. They discussed the maximum likelihood estimation of the model parameters. The probability density function of Weibull Lomax distribution is given by

$$
f(x ; \theta)=\frac{a b \theta}{\lambda}\left[1+\frac{x}{\lambda}\right]^{a b-1}\left[1-\left(1+\frac{x}{\lambda}\right)^{-a}\right]^{b-1} e^{-\theta\left[\left(1+\frac{x}{\lambda}\right)^{a}-1\right]^{b}} ; x>0 .
$$

The joint density function or likelihood function of (1) is given by

$$
f(\underline{x} ; \theta)=\left(\frac{a b \theta}{\lambda}\right)^{n}\left(\prod_{i=1}^{n}\left[1+\frac{x_{i}}{\lambda}\right]^{a b-1}\left[1-\left(1+\frac{x_{i}}{\lambda}\right)^{-a}\right]^{b-1}\right) e^{-\theta \sum_{i=1}^{n}\left[\left(1+\frac{x}{\lambda}\right)^{a}-1\right]^{b}} .
$$

The log likelihood function is given by

$\left.\log f_{X}, \theta\right)=n \log \left(\frac{a b \theta}{\lambda}\right)+\log \left(\prod_{i=1}^{n}\left[1+\frac{x_{i}}{\lambda}\right]^{a b-1}\left[1-\left(1+\frac{x_{i}}{\lambda}\right)^{-a}\right]^{b-1}\right)-\theta \sum_{i=1}^{n}\left[\left(1+\frac{x}{\lambda}\right)^{a}-1\right]^{b}$ 
Differentiating (3) with respect to $\theta$ and equating to zero, we get the maximum likelihood estimator of $\theta$ which is given as

$$
\hat{\theta}=n\left(\sum_{i=1}^{n}\left[\left(1+\frac{x}{\lambda}\right)^{a}-1\right]^{b}\right)^{-1} .
$$

\section{Bayesian Method of Estimation}

The Bayesian inference procedures have been developed generally under squared error loss function

$$
L(\hat{\theta}, \theta)=(\hat{\theta}-\theta)^{2}
$$

The Bayes estimator under the above loss function, say, $\hat{\theta}_{s}$ is the posterior mean, i.e,

$$
\hat{\theta}_{S}=E(\theta) .
$$

Zellner [2], Basu and Ebrahimi [3] have recognized that the inappropriateness of using symmetric loss function. Norstrom [4] introduced precautionary loss function is given as

$$
L(\hat{\theta}, \theta)=\frac{(\hat{\theta}-\theta)^{2}}{\hat{\theta}} .
$$

The Bayes estimator under this loss function is denoted by $\hat{\theta}_{P}$ and is obtained as

$$
\hat{\theta}_{P}=\left[E\left(\theta^{2}\right)\right]^{1 / 2} \text {. }
$$

Calabria and Pulcini [5] points out that a useful asymmetric loss function is the entropy loss

$$
L(\delta) \propto\left[\delta^{p}-p \log _{e}(\delta)-1\right]
$$

where $\delta=\frac{\hat{\theta}}{\theta}$, and whose minimum occurs at $\hat{\theta}=\theta$. Also, the loss function $L(\delta)$ has been used in Dey et al. [6] and Dey and Liu [7], in the original form having $p=1$. Thus $L(\delta)$ can written be as 


$$
L(\delta)=b\left[\delta-\log _{e}(\delta)-1\right] ; b>0 .
$$

The Bayes estimator under entropy loss function is denoted by $\hat{\theta}_{E}$ and is obtained by solving the following equation

$$
\hat{\theta}_{E}=\left[E\left(\frac{1}{\theta}\right)\right]^{-1} .
$$

Wasan [8] proposed the K-loss function which is given as

$$
L(\hat{\theta}, \theta)=\frac{(\hat{\theta}-\theta)^{2}}{\hat{\theta} \theta} .
$$

Under K-loss function the Bayes estimator of $\theta$ is denoted by $\hat{\theta}_{K}$ and is obtained as

$$
\hat{\theta}_{K}=\left[\frac{E(\theta)}{E(1 / \theta)}\right]^{\frac{1}{2}} .
$$

Al-Bayyati [9] introduced a new loss function which is given as

$$
L(\hat{\theta}, \theta)=\theta^{c}(\hat{\theta}-\theta)^{2}
$$

Under Al-Bayyati's loss function the Bayes estimator of $\theta$ is denoted by $\hat{\theta}_{A l}$ and is obtained as

$$
\hat{\theta}_{A l}=\frac{E\left(\theta^{c+1}\right)}{E\left(\theta^{c}\right)} .
$$

Let us consider two prior distributions of $\theta$ to obtain the Bayes estimators.

(i) Quasi-prior: For the situation where we have no prior information about the parameter $\theta$, we may use the quasi density as given by

$$
g_{1}(\theta)=\frac{1}{\theta^{d}} ; \theta>0, d \geq 0,
$$

where $d=0$ leads to a diffuse prior and $d=1$, a non-informative prior. 
(ii) Gamma prior: Generally, the gamma density is used as prior distribution of the parameter $\theta$ given by

$$
g_{2}(\theta)=\frac{\beta^{\alpha}}{\Gamma(\alpha)} \theta^{\alpha-1} e^{-\beta \theta} ; \theta>0
$$

\section{Posterior Density under $g_{1}(\theta)$}

The posterior density of $\theta$ under $g_{1}(\theta)$, on using (2), is given by

$$
\begin{aligned}
f(\theta / \underline{x})= & \frac{\left(\frac{a b \theta}{\lambda}\right)^{n}\left(\prod_{i=1}^{n}\left[1+\frac{x_{i}}{\lambda}\right]^{a b-1}\left[1-\left(1+\frac{x_{i}}{\lambda}\right)^{-a}\right]^{b-1}\right) e^{-\theta \sum_{i=1}^{n}\left[\left(1+\frac{x}{\lambda}\right)^{a}-1\right]^{b}} \theta^{-d}}{\int_{0}^{\infty}\left(\frac{a b \theta}{\lambda}\right)^{n}\left(\prod_{i=1}^{n}\left[1+\frac{x_{i}}{\lambda}\right]^{a b-1}\left[1-\left(1+\frac{x_{i}}{\lambda}\right)^{-a}\right]^{b-1}\right) e^{-\theta \sum_{i=1}^{n}\left[\left(1+\frac{x}{\lambda}\right)^{a}-1\right]} \theta^{-d} d \theta} \\
= & \frac{\theta^{n-d} e^{-\theta \sum_{i=1}^{n}\left[\left(1+\frac{x}{\lambda}\right)^{a}-1\right]^{b}}}{\int_{0}^{\infty} \theta^{n-d} e^{-\theta \sum_{i=1}^{n}\left[\left(1+\frac{x}{\lambda}\right)^{a}-1\right]^{b}} d \theta} \\
= & \frac{\left(\sum_{i=1}^{n}\left[\left(1+\frac{x}{\lambda}\right)^{a}-1\right]^{b}\right)^{n-d+1}}{\Gamma(n-d+1)} \theta^{n-d} e^{-\theta \sum_{i=1}^{n}\left[\left(1+\frac{x}{\lambda}\right)^{a}-1\right]}
\end{aligned}
$$

Theorem 1. On using (17), we have

$$
E\left(\theta^{c}\right)=\frac{\Gamma(n-d+c+1)}{\Gamma(n-d+1)}\left(\sum_{i=1}^{n}\left[\left(1+\frac{x}{\lambda}\right)^{a}-1\right]^{b}\right)^{-c} .
$$

Proof. By definition,

$$
E\left(\theta^{c}\right)=\int \theta^{c} f(\theta / \underline{x}) d \theta
$$




$$
\begin{aligned}
& =\frac{\left(\sum_{i=1}^{n}\left[\left(1+\frac{x}{\lambda}\right)^{a}-1\right]^{b}\right)^{n-d+1}}{\Gamma(n-d+1)} \int_{0}^{\infty} \theta^{(n-d+c)} e^{-\theta \sum_{i=1}^{n}\left[\left(1+\frac{x}{\lambda}\right)^{a}-1\right]^{b}} d \theta \\
& =\frac{\left(\sum_{i=1}^{n}\left[\left(1+\frac{x}{\lambda}\right)^{a}-1\right]^{b}\right)^{n-d+1}}{\Gamma(n-d+1)} \frac{\Gamma(n-d+c+1)}{\left(\sum_{i=1}^{n}\left[\left(1+\frac{x}{\lambda}\right)^{a}-1\right]^{b}\right)^{n-d+c+1}} \\
& =\frac{\Gamma(n-d+c+1)}{\Gamma(n-d+1)}\left(\sum_{i=1}^{n}\left[\left(1+\frac{x}{\lambda}\right)^{a}-1\right]^{b}\right)^{-c} .
\end{aligned}
$$

From equation (18), for $c=1$, we have

$$
E(\theta)=(n-d+1)\left(\sum_{i=1}^{n}\left[\left(1+\frac{x}{\lambda}\right)^{a}-1\right]^{b}\right)^{-1}
$$

From equation (18), for $c=2$, we have

$$
E\left(\theta^{2}\right)=[(n-d+2)(n-d+1)]\left[\sum_{i=1}^{n}\left[\left(1+\frac{x}{\lambda}\right)^{a}-1\right]^{b}\right]^{-2}
$$

From equation (18), for $c=-1$, we have

$$
E\left(\frac{1}{\theta}\right)=\frac{1}{(n-d)} \sum_{i=1}^{n}\left[\left(1+\frac{x}{\lambda}\right)^{a}-1\right]^{b} .
$$

From equation (18), for $c=c+1$, we have

$$
E\left(\theta^{c+1}\right)=\frac{\Gamma(n-d+c+2)}{\Gamma(n-d+1)}\left(\sum_{i=1}^{n}\left[\left(1+\frac{x}{\lambda}\right)^{a}-1\right]^{b}\right)^{-(c+1)}
$$




\section{Bayes Estimators under $g_{1}(\theta)$}

From equation (6), on using (19), the Bayes estimator of $\theta$ under squared error loss function is given by

$$
\hat{\theta}_{S}=(n-d+1)\left(\sum_{i=1}^{n}\left[\left(1+\frac{x}{\lambda}\right)^{a}-1\right]^{b}\right)^{-1}
$$

From equation (8), on using (20), the Bayes estimator of $\theta$ under precautionary loss function is obtained as

$$
\hat{\theta}_{P}=[(n-d+2)(n-d+1)]^{\frac{1}{2}}\left(\sum_{i=1}^{n}\left[\left(1+\frac{x}{\lambda}\right)^{a}-1\right]^{b}\right)^{-1}
$$

From equation (10), on using (21), the Bayes estimator of $\theta$ under entropy loss function is given by

$$
\hat{\theta}_{E}=(n-d)\left(\sum_{i=1}^{n}\left[\left(1+\frac{x}{\lambda}\right)^{a}-1\right]^{b}\right)^{-1}
$$

From equation (12), on using (19) and (21), the Bayes estimator of $\theta$ under K-loss function is given by

$$
\hat{\theta}_{K}=[(n-d+1)(n-d)]^{\frac{1}{2}}\left(\sum_{i=1}^{n}\left[\left(1+\frac{x}{\lambda}\right)^{a}-1\right]^{b}\right)^{-1} .
$$

From equation (14), on using (18) and (22), the Bayes estimator of $\theta$ under Al-Bayyati's loss function comes out to be

$$
\hat{\theta}_{A l}=(n-d+c+1)\left(\sum_{i=1}^{n}\left[\left(1+\frac{x}{\lambda}\right)^{a}-1\right]^{b}\right)^{-1} .
$$

\section{Posterior Density under $g_{2}(\theta)$}

Under $g_{2}(\theta)$, the posterior density of $\theta$, using equation (2), is obtained as 
Estimation of Scale Parameter of Weibull-Lomax Distribution via Bayesian Approach

$$
\begin{aligned}
& f(\theta / \underline{x})=\frac{\left(\frac{a b \theta}{\lambda}\right)^{n}\left(\prod_{i=1}^{n}\left[1+\frac{x_{i}}{\lambda}\right]^{a b-1}\left[1-\left(1+\frac{x_{i}}{\lambda}\right)^{-a}\right]^{b-1}\right) e^{-\theta \sum_{i=1}^{n}\left[\left(1+\frac{x}{\lambda}\right)^{a}-1\right]^{b}} \frac{\beta^{\alpha}}{\Gamma(\alpha)} \theta^{\alpha-1} e^{-\beta \theta}}{\int_{0}^{\infty}\left(\frac{a b \theta}{\lambda}\right)^{n}\left(\prod_{i=1}^{n}\left[1+\frac{x_{i}}{\lambda}\right]^{a b-1}\left[1-\left(1+\frac{x_{i}}{\lambda}\right)^{-a}\right]^{b-1}\right) e^{-\theta \sum_{i=1}^{n}\left[\left(1+\frac{x}{\lambda}\right)^{a}-1\right]^{b}} \frac{\beta^{\alpha}}{\Gamma(\alpha)} \theta^{\alpha-1} e^{-\beta \theta} d \theta} \\
& =\frac{\theta^{n+\alpha-1} e^{\left.-\left(\beta+\sum_{i=1}^{n}\left[\left(1+\frac{x}{\lambda}\right)^{a}-1\right]\right]^{b}\right) \theta}}{\int_{0}^{\infty} \theta^{n+\alpha-1} e^{\left.-\left(\beta+\sum_{i=1}^{n}\left[\left(1+\frac{x}{\lambda}\right)^{a}-1\right]\right]^{b}\right) \theta} d \theta} \\
& =\frac{\theta^{n+\alpha-1} e^{\left.-\left(\beta+\sum_{i=1}^{n}\left[\left(1+\frac{x}{\lambda}\right)^{a}-1\right]\right]^{b}\right) \theta}}{\Gamma(n+\alpha) /\left(\beta+\sum_{i=1}^{n}\left[\left(1+\frac{x}{\lambda}\right)^{a}-1\right]^{b}\right)^{n+\alpha}} \\
& =\frac{\left(\beta+\sum_{i=1}^{n}\left[\left(1+\frac{x}{\lambda}\right)^{a}-1\right]^{b}\right)^{n+\alpha}}{\Gamma(n+\alpha)} \theta^{n+\alpha-1} e^{\left.-\left(\beta+\sum_{i=1}^{n}\left[\left(1+\frac{x}{\lambda}\right)^{a}-1\right]\right]^{b}\right) \theta}
\end{aligned}
$$

Theorem 2. On using (28), we have

$$
E\left(\theta^{c}\right)=\frac{\Gamma(n+\alpha+c)}{\Gamma(n+\alpha)}\left(\beta+\sum_{i=1}^{n}\left[\left(1+\frac{x}{\lambda}\right)^{a}-1\right]^{b}\right)^{-c}
$$

Proof. By definition,

$$
\begin{aligned}
E\left(\theta^{c}\right) & =\int \theta^{c} f(\theta / \underline{x}) d \theta \\
& =\frac{\left(\beta+\sum_{i=1}^{n}\left[\left(1+\frac{x}{\lambda}\right)^{a}-1\right]^{b}\right)^{n+\alpha}}{\Gamma(n+\alpha)} \int_{0}^{\infty} \theta^{n+\alpha+c-1} e^{-\left(\beta+\sum_{i=1}^{n}\left[\left(1+\frac{x}{\lambda}\right)^{a}-1\right]^{b}\right) \theta} d \theta
\end{aligned}
$$




$$
\begin{aligned}
& =\frac{\left(\beta+\sum_{i=1}^{n}\left[\left(1+\frac{x}{\lambda}\right)^{a}-1\right]^{b}\right)^{n+\alpha}}{\Gamma(n+\alpha)} \frac{\Gamma(n+\alpha+c)}{\left(\beta+\sum_{i=1}^{n}\left[\left(1+\frac{x}{\lambda}\right)^{a}-1\right]^{b}\right)^{n+\alpha+c}} \\
& =\frac{\Gamma(n+\alpha+c)}{\Gamma(n+\alpha)}\left(\beta+\sum_{i=1}^{n}\left[\left(1+\frac{x}{\lambda}\right)^{a}-1\right]^{b}\right)^{-c} .
\end{aligned}
$$

From equation (29), for $c=1$, we have

$$
E(\theta)=(n+\alpha)\left(\beta+\sum_{i=1}^{n}\left[\left(1+\frac{x}{\lambda}\right)^{a}-1\right]^{b}\right)^{-1} .
$$

From equation (29), for $c=2$, we have

$$
E\left(\theta^{2}\right)=[(n+\alpha+1)(n+\alpha)]\left(\beta+\sum_{i=1}^{n}\left[\left(1+\frac{x}{\lambda}\right)^{a}-1\right]^{b}\right)^{-2} .
$$

From equation (29), for $c=-1$, we have

$$
E\left(\frac{1}{\theta}\right)=\frac{1}{(n+\alpha-1)}\left(\beta+\sum_{i=1}^{n}\left[\left(1+\frac{x}{\lambda}\right)^{a}-1\right]^{b}\right) .
$$

From equation (29), for $c=c+1$, we have

$$
E\left(\theta^{c+1}\right)=\frac{\Gamma(n+\alpha+c+1)}{\Gamma(n+\alpha)}\left(\beta+\sum_{i=1}^{n}\left[\left(1+\frac{x}{\lambda}\right)^{a}-1\right]^{b}\right)^{-(c+1)} .
$$

\section{Bayes Estimators under $g_{2}(\theta)$}

From equation (6), on using (30), the Bayes estimator of $\theta$ under squared error loss function is given by 


$$
\hat{\theta}_{S}=(n+\alpha)\left(\beta+\sum_{i=1}^{n}\left[\left(1+\frac{x}{\lambda}\right)^{a}-1\right]^{b}\right)^{-1} .
$$

From equation (8), on using (31), the Bayes estimator of $\theta$ under precautionary loss function is obtained as

$$
\hat{\theta}_{P}=[(n+\alpha+1)(n+\alpha)]^{\frac{1}{2}}\left(\beta+\sum_{i=1}^{n}\left[\left(1+\frac{x}{\lambda}\right)^{a}-1\right]^{b}\right)^{-1} .
$$

From equation (10), on using (32), the Bayes estimator of $\theta$ under entropy loss function is given by

$$
\hat{\theta}_{E}=(n+\alpha+1)\left(\beta+\sum_{i=1}^{n}\left[\left(1+\frac{x}{\lambda}\right)^{a}-1\right]^{b}\right)^{-1}
$$

From equation (12), on using (30) and (32), the Bayes estimator of $\theta$ under K-loss function is given by

$$
\hat{\theta}_{K}=[(n+\alpha)(n+\alpha-1)]^{\frac{1}{2}}\left(\beta+\sum_{i=1}^{n}\left[\left(1+\frac{x}{\lambda}\right)^{a}-1\right]^{b}\right)^{-1} \quad .
$$

From equation (14), on using (29) and (33), the Bayes estimator of $\theta$ under Al-Bayyati's loss function comes out to be

$$
\hat{\theta}_{A l}=(n+\alpha+c)\left(\beta+\sum_{i=1}^{n}\left[\left(1+\frac{x}{\lambda}\right)^{a}-1\right]^{b}\right)^{-1} .
$$

\section{Conclusion}

In this paper, we have obtained a number of estimators of parameter of Weibull-Lomax distribution. In equation (4) we have obtained the maximum likelihood estimator of the parameter. In equation (23), (24), (25), (26) and (27) we have obtained the Bayes estimators under different loss functions using quasi prior. In equation (34), (35), (36), (37) and (38) we have obtained the Bayes estimators under different loss functions using gamma prior. In the above equation, it is clear that the Bayes estimators depend upon the parameters of the prior distribution. We therefore recommend that the estimator's choice lies according to the value of the prior distribution which in turn depends on the situation at hand. 


\section{References}

[1] Tahir, M.H, Cordeiro, G.M. and Zubair, M., (2015): "The Weibull-Lomax distribution: properties and applications". Hacettepe Journal of Mathematics and Statistics, Vol. 44(2), 461480.

[2] Zellner, A., (1986): "Bayesian estimation and prediction using asymmetric loss functions". Jour. Amer. Stat. Assoc., 91, 446-451.

[3] Basu, A. P. and Ebrahimi, N., (1991): "Bayesian approach to life testing and reliability estimation using asymmetric loss function". Jour. Stat. Plann. Infer., 29, 21-31.

[4] Norstrom, J. G., (1996): “The use of precautionary loss functions in Risk Analysis". IEEE Trans. Reliab., 45(3), 400-403.

[5] Calabria, R., and Pulcini, G. (1994): "Point estimation under asymmetric loss functions for left truncated exponential samples". Comm. Statist. Theory \& Methods, 25 (3), 585-600.

[6] D.K. Dey, M. Ghosh and C. Srinivasan (1987): "Simultaneous estimation of parameters under entropy loss". Jour. Statist. Plan. And infer., 347-363.

[7] D.K. Dey, and Pei-San Liao Liu (1992): "On comparison of estimators in a generalized life Model”. Microelectron. Reliab. 32 (1/2), 207-221.

[8] Wasan, M.T., (1970): "Parametric Estimation”. New York: Mcgraw-Hill.

[9] Al-Bayyati, (2002): "Comparing methods of estimating Weibull failure models using simulation". Ph.D. Thesis, College of Administration and Economics, Baghdad University, Iraq. 